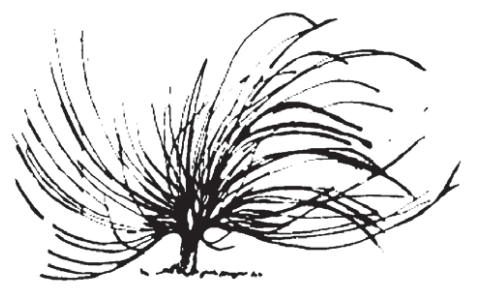

\title{
Conductismo vs. Constructivismo: Sus Principales Aportes en la Pedagogía, el Diseño Curricular e Intruccional en el Área de las Ciencias Naturales
}

\author{
Rosita Ulate Sánchez ${ }^{1}$ \\ Universidad Estatal a Distancia \\ San José, Costa Rica \\ rulate@nova.edu
}

\begin{abstract}
Resumen
El presente ensayo fue preparado como parte de una reflexión de la autora en el curso de Introducción al "Diseño Instruccional" en el Programa de Doctorado en Educación en Nova Southeastern University. El tema nació de la necesidad de comparar dos enfoques de las teorías de aprendizaje, el Conductismo vs. Constructivismo, sus aportes en la pedagogía y consecuentemente en el diseño curricular e instruccional. Se logró una aproximación a los fundamentos epistemológicos del conductismo y del constructivismo, su relación con la pedagogía utilizada en el área de las ciencias naturales y finalmente un análisis de la contribución de ambas posturas teóricas del aprendizaje con el diseño curricular y el diseño instruccional de la carrera en estudio.
\end{abstract}

Recibido: 17 de setiembre de 2013 - Aprobado: 12 de febrero de 2014

1 Rosita Ulate, realizó sus estudios de pre grado en la Carrera de Administración de Empresas, así como su Maestría en esta misma rama de la administración con Énfasis en Mercadeo en la Fundación de Estudios de Pos grado de la Universidad de Costa Rica; actualmente es egresada del Doctorado en Educación a Distancia en Tecnología Instruccional de NOVA Southeastern University en Florida, Estados Unidos. 
Palabras claves: teorías del aprendizaje, conductismo, constructivismo, manejo de recursos naturales, diseño curricular, diseño instruccional, pedagogía

\begin{abstract}
This essay was prepared as part of a reflection of the author in the course "Introduction to Instructional Design" in the Doctoral Program in Education at Nova Southeastern University. The topic addressed arose from the need to compare two approaches to learning theories, behaviorism vs. constructivism and their contributions to pedagogy and consequently to curricular and instructional design. An approximation to the epistemological foundations of behaviorism and constructivism and their connection with the pedagogy used in the area of natural sciences was achieved. Finally, an analysis of the contribution of both theoretical positions of learning with curriculum design and instructional design in natural sciences was made.
\end{abstract}

Keywords: learning theories, behaviorism, constructivism, natural resource management, curriculum design, instructional design, pedagogy

\title{
Introducción
}

U

n paradigma se puede definir como un conjunto de creencias

y principios bajo los cuales se estudia un fenómeno natural a partir de una serie de supuestos ontológicos, metodológicos y epistemológicos (Guba, 1990). Los paradigmas son asumidos como enfoques para conocer e interpretar aquellos criterios que componen la explicación de un hecho o un suceso.

De acuerdo con Martín (1991), la epistemología busca estudiar el origen y validez del saber y puede ser enfocada desde la teoría de la ciencia o del conocimiento. Por lo que puede ser entendida desde el modelo de la relación entre el investigador y el objeto investigado o desde la explicación del cómo se llega a un conocimiento determinado.

Schunk (1997) indica que la epistemología explica el origen, la naturaleza, los límites y métodos del conocimiento por medio del aprendizaje. Por lo anterior el establecimiento de los fundamentos epistemológicos de las teorías de aprendizaje son de vital importancia para 
los involucrados en el ámbito educativo. Razón por la cual se realiza un estudio descriptivo y comparativo de dos teorías del aprendizaje como lo son el conductismo y el constructivismo.

Este ensayo se divide en varios apartados, en primer lugar, se presenta la definición, fundamentos epistemológicos y aportes a la pedagogía de cada una de las dos teorías de aprendizaje escogidas. En segundo lugar, se hace una presentación de los principales fundamentos y exponentes de las teorías de aprendizaje. En tercer lugar, se presentan los mayores aportes de las dos teorías de aprendizaje al diseño curricular e instruccional para terminar en un cuarto aspecto con el análisis desde la enseñanza de las ciencias naturales.

\section{Fundamentos epistemológicos del conductismo y del constructivismo}

Desde el enfoque del realismo se pueden comprender las raíces filosóficas y las bases ideológicas del conocimiento asociado a la teoría conductista. A partir de la concepción del realismo el investigador puede conocer una verdad que existe, sin provocar una interrelación con su objeto de estudio. A partir de este principio, el conductismo se complementa con tres de las principales direcciones paradigmáticas, como lo son el empirismo, el positivismo y el pragmatismo.

El empirismo, como epistemología del positivismo, explica la adquisición del conocimiento, tal y como lo llamó Aristóteles: un mismo punto de partida para la adquisición del conocimiento por medio de la experiencia en el contexto. Conocimiento que luego expresado en un criterio de verdad, podrá ser aceptado o rechazado por otros. Esa secuencia de pasos llegó a convertirse en el pilar del método científico. De tal forma que todo conocimiento se adquiere por la comparación y el descubrimiento de relaciones entre los objetos de estudio, ideas o cuestiones de hecho. Por su parte la objetividad del positivismo asumió como únicos medios del conocimiento: la ciencia y su método científico. Esta corriente se concentró en la descripción de los hechos observables, ajenos a los intereses del investigador y por medio de procesos generalizados, así como de inducción. Claves maestras para establecer relaciones convertidas en leyes, con lo que se llegaban a explicar los fenómenos naturales.

Los aspectos del empirismo y del positivismo son el preámbulo para que desde el pragmatismo se promulgue que lo único útil es la verdad. De acuerdo con Driscoll (2005), dentro de las tradiciones 
epistemológicas del aprendizaje el conductismo asume la realidad objetiva, singular y fragmentada.

El conductismo permite la observación de la interacción entre la conducta del objeto de investigación con los eventos en su ambiente. Este fenómeno es analizado y traducido a criterios observables, medibles y que puedan ser reproducibles.

Por otra parte, la teoría del constructivismo también cuenta con una serie de aportes que datan desde los tiempos de los filósofos griegos, para lo cual se remite a los versos de Jenófanes citado por López (s.f.). Al respecto López (s.f.) menciona que Protágoras abandonó la objetividad para expresar "En todas las cosas hay dos razones contrarias entre sí (...) el hombre es la medida de todas las cosas" (p.3). El mismo autor, presenta al hombre como constructor de la realidad y de la subjetividad, cuando expone la importancia del contexto social asumiendo que "el parecer de la colectividad es el que se hace verdadero cuando se formula y durante todo el tiempo que dure ese parecer" (p.4).

El constructivismo desde una posición filosófica se apoya ontológicamente en el pensamiento de la crítica, que da paso al relativismo y desde la epistemología a las discusiones críticas al subjetivismo. La misma teoría crítica propone que el conocimiento es formado por las estructuras cognitivas no pasivas y sucesivas del sujeto, como producto de las experiencias individuales dentro de un contexto social, aspectos que dan base al movimiento constructivista.

Desde la posición del enfoque constructivista el investigador u observador no es ajeno a sus valores propios en el proceso de investigación y por lo tanto la construcción del conocimiento está dentro de un entorno social y biológico. De tal forma que el objeto de estudio y el investigador están interrelacionados, y por lo tanto, el conocimiento se construye en el proceso (Guba y Lincoln, 1989 citado por Velandia 2006). El cambio de visión de la relación investigador y objeto de estudio es conocido como el rompimiento y el nacimiento de las diferencias entre la ontología y la epistemología. Razón por la cual Bustos Cobos (1994) mencionado por De Zubiría (2008) llaman a los constructivistas "hijos del Estructuralismo Piagetiano y nietos de la Filosofía Kantiana" (p.155); destacando que el constructivismo es el inicio de un nuevo planteamiento epistemológico.

El constructivismo como nuevo paradigma se apoya en varias disciplinas, tales como la psicología cognitiva y humanista, la filosofía 
y la educación. Este nuevo movimiento promovió el abandono de la concepción epistemológica realista o empirista y asociativa, para explicar el desarrollo humano desde la subjetividad. De acuerdo con Driscoll (2005), desde el interpretismo en donde se asume una realidad holística y múltiple. Por lo tanto, el constructivismo es una interpretación de la realidad basada en el aporte de varias disciplinas.

\section{Precursores y Exponentes}

Son varias las iniciativas epistemológicas que se encuentran alrededor del conductismo y del constructivismo, razón por la cual se considera relevante la presentación a manera de repaso de los principales precursores y exponentes de ambos puntos de vista paradigmáticos. En la Tabla 1, se observa el empirismo, el positivismo y el pragmatismo, que son fuentes precursoras epistemológicamente del conductismo a través del seguimiento del método inductivo, la relación de causa y efecto y la experiencia empírica, posiciones implementadas especialmente en las ciencias naturales. Mientras que desde el interpretismo se aboga por una activa construcción del conocimiento y la interrelación con el contexto en el cual se desarrolle el ser humano. 


\section{Tabla 1: Precursores epistemológicos del} conductismo y constructivismo

\begin{tabular}{|c|c|c|}
\hline Conductismo & $\begin{array}{l}\text { Positivismo } \\
\text { Pragmatismo }\end{array}$ & $\begin{array}{l}\text { En el siglo XVI Francis Bacon expone } \\
\text { el método inductivo en los procesos de } \\
\text { aprendizaje. Manifestó que la mente de un } \\
\text { individuo esta como una hoja en blanco, } \\
\text { en la cual la experiencia imprime el } \\
\text { conocimiento (Cattaneo, s.f.; Hernández, } \\
\text { 2008 y Soto y Bernardini, 2007). } \\
\text { David Hume rechaza la idea de la } \\
\text { causalidad. Ve el conocimiento de los } \\
\text { hechos como imposible aunque acepta el } \\
\text { pensamiento de causa-efecto y las relaciones } \\
\text { entre las ideas (Catanneo, s.f.) } \\
\text { George Berckely, al igual que Hume, negaba } \\
\text { la existencia de la parte espiritual (Soto y } \\
\text { Bernardini, 2007). } \\
\text { Augusto Comté propuso abordar los } \\
\text { fenómenos sociales desde la lógica de la } \\
\text { investigación de las ciencias naturales } \\
\text { (Salas, 2002). } \\
\text { John Locke recomendó el método de } \\
\text { educación y el currículo, el cual se basaba } \\
\text { en un la experiencia empírica antes de llegar } \\
\text { a conclusiones. Su pensamiento fue de gran } \\
\text { influencia en los educadores de los siglos } \\
\text { XVII y XVIII, Cattaneo (s.f.). }\end{array}$ \\
\hline Constructivismo & Interpretismo & $\begin{array}{l}\text { En el siglo XVII Giambattista Vico acentúa } \\
\text { el saber como una activa construcción del } \\
\text { ser humano (Soto y Bernardini, } 2007 \text { y } \\
\text { López, s.f.). } \\
\text { John Dewey vincula la filosofía con la } \\
\text { pedagogía. Aboga por la reconstrucción } \\
\text { continua y reorganización de las } \\
\text { experiencias dentro de un sentido moral a } \\
\text { la ayuda personal y de la sociedad (Soto y } \\
\text { Bernardini, 2007). }\end{array}$ \\
\hline
\end{tabular}

Fuente: Catataneo (s.f); Hernández, (2008); Soto y Bernardini, (2007) y Salas, (2002).

Los aportes de varias disciplinas se conjugan para influenciar y construir las teorías del aprendizaje que son asumidas por varios exponentes del conductismo y el constructivismo en las teorías del aprendizaje. En la Tabla 2, se observan los principales aportes de los grandes exponentes del conductismo, entre ellos Pavlov, Watson y Skinner. 
Mientras que dentro de los padres de la teoría de aprendizaje constructivista se encuentran Peaget, Vygostsky, Gagné (más asociado con el cognitivismo), Ausubel, Anderson, Novak, Kelly, así como Von Glaserfiel.

\section{Tabla 2: Exponentes del conductismo y constructivismo}

\begin{tabular}{|c|c|}
\hline Teorías & Expositores y algunos aportes de acuerdo a varios autores \\
\hline Conductismo & $\begin{array}{l}\text { - Iván Pavlov inicia los estudios que condujeron a las bases del } \\
\text { conductismo. Se le atribuye el condicionamiento clásico. Se destaca } \\
\text { las acciones menos complejas de los reflejos. Fundamento para el } \\
\text { trabajo que realizó más tarde J. Watson (Driscoll, 2005). } \\
\text { James Watson, retoma los estudios de Pavlov y llega a dominar las } \\
\text { teorías del aprendizaje estadounidense. Indico que la conducta puede } \\
\text { ser cuantificable y medible objetivamente (Driscoll, 2005). } \\
\text { Frederic Skinner exponente del conductismo radical y del } \\
\text { condicionamiento operativo. Propone el análisis experimental del } \\
\text { comportamiento como medio para relacionar variables ambientales } \\
\text { y conductuales. Los conceptos de asociación permearon las teorías } \\
\text { de aprendizaje (Driscoll, 2005). }\end{array}$ \\
\hline Constructivismo & $\begin{array}{l}\text { - Jean Piaget trabaja las ideas de asimilación y acomodación. } \\
\text { Representante del constructivismo llamado como Piagetiano en su } \\
\text { honor. Sigue desde la epistemología evolutiva y se traduce en la } \\
\text { construcción del conocimiento de acuerdo con las etapas psico - } \\
\text { evolutiva de los niños. Tuvo gran impacto en la psicología infantil y } \\
\text { de la educación (Cattaneo, s.f.; De Zubiría, 2008 y Thanaoulas, s.f.). } \\
\text { Lev Vygostsky como precursor del constructivismo social, observa al } \\
\text { individuo como un producto de su historia y de su contexto social y } \\
\text { cultural en donde el lenguaje juega un papel importante (Driscoll, 2005). } \\
\text { Robert Gagné con sus aportes del aprendizaje previo y Brunner con las } \\
\text { ideas de asociación como eje central del conocimiento (Thanasoulas, s.f.). } \\
\text { David Ausubel expone la importancia de los puentes y relaciones } \\
\text { cognitivas. Al que han llamado el constructivismo humano por sus } \\
\text { aportes del aprendizaje significativo (Cattaneo, s.f. y Driscoll, 2005). } \\
\text { Anderson y su teoría de esquemas así como Novak, por sus } \\
\text { contribuciones de la neurobiológica en la construcción del } \\
\text { conocimiento (Driscoll, 2005). } \\
\text { George Kelly y el llamado constructivismo social, con fundamento en } \\
\text { la importancia de las ideas alternativas y el cambio conceptual, a partir } \\
\text { de las teorías del procesamiento de la información (Cattaneo, s.f.). } \\
\text { Von Glaserfeld y el constructivismo radical, que rechaza o niega la } \\
\text { posibilidad de la transmisión entre el profesor y el estudiante, por } \\
\text { ser construcciones individuales de cada uno, con gran influencia en } \\
\text { la construcción del conocimiento de Charles Darwin y del proceso } \\
\text { cognitivo. Como la adaptación al medio por un descubrimiento de la } \\
\text { realidad objetiva (Cattaneo, s.f.). }\end{array}$ \\
\hline
\end{tabular}

Fuente: Cattaneo, (s.f.); De Zubriria, (2008); Driscoll, (2005) y Thanaoulas, (s.f.). 


\section{Aportes del conductismo y el constructivismo a la pedagogía}

De acuerdo con Salas (2002), "el propósito del conductismo no es otro que moldear la conducta a través de la manipulación intencionada del ambiente" (p.13). La misma autora distingue los aportes del constructivismo a la tecnología educativa indicando que "elabora didácticas que permiten el logro de procesos cognitivos en donde lo subjetivo - construcción de significados- $y$ la investigación - conocimiento desarrollado por el propio sujeto-, son fundamentos a nivel curricular", con evaluación cualitativa y cuantitativa" (p.15), e indica que el conductismo es la base de la pedagogía educativa conductista que:

elabora didáctica que permiten el logro de objetivos determinados (...) además, como su método de enseñanza se basa en objetivos, éstos pueden ser evaluados en forma cuantitativa y de manera progresiva yendo de lo más simple hasta lo más complejo. (pp. 13-14).

De tal forma, que desde la diferentes perspectivas de la pedagogía, ésta brinda bases y lineamientos para estructurar y analizar la educación en su proceso de enseñanza y aprendizaje, ya sea desde el conductivismo o el constructivismo, aspectos que influyen en el diseño curricular e instruccional. La pedagogía en la teoría de aprendizaje conductista, se enfoca en una didáctica que apoya la enseñanza moldeadora de la conducta de los estudiantes, utilizando el diseño del currículo por objetivos y predomina la evaluación cuantitativa del rendimiento académico (véase Figura 1). 
Figura 1: Aportes del conductismo a la pedagogía

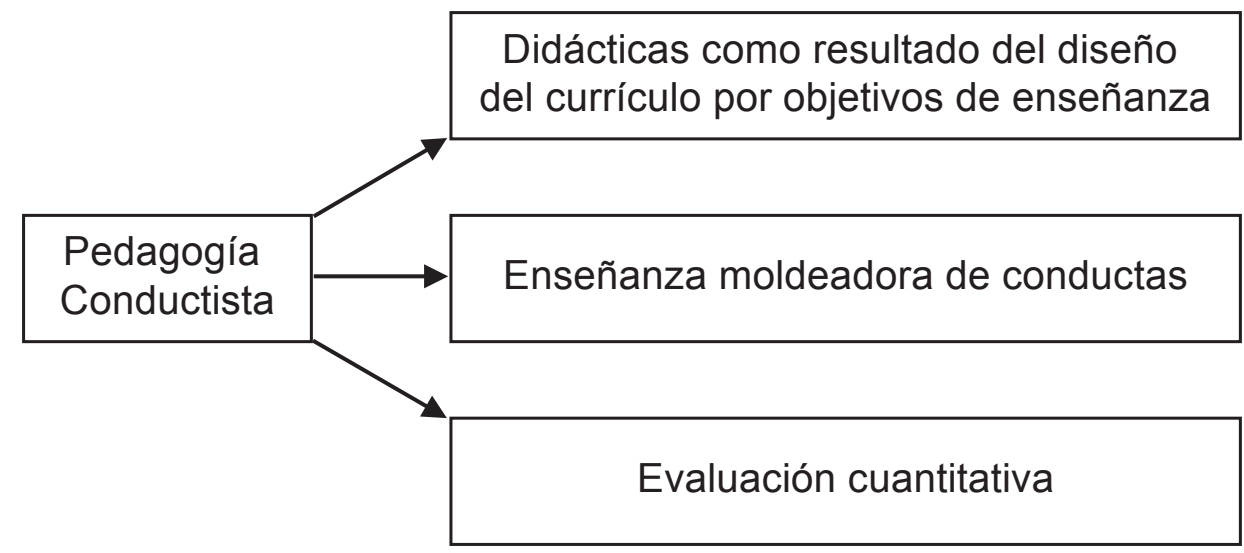

Adaptado de Salas (2002, p.13).

En la Figura 2, se visualiza, como por su parte la pedagogía bajo el enfoque de la teoría de aprendizaje del constructivismo se basa en diseño de currículo que abarca procesos tanto cognitivos como subjetivos, en donde se busca una evaluación integral del proceso de aprendizaje bajo una enseñanza constructora entre los sujetos involucrados.

Figura 2: Aportes del constructivismo a la pedagogía

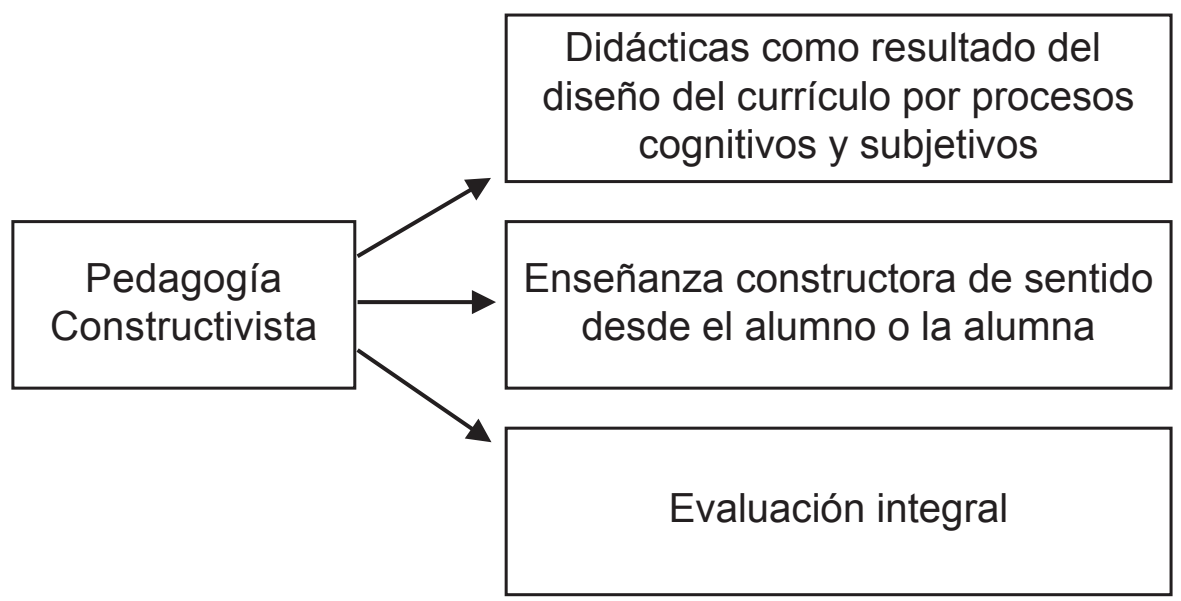

Adaptado de Salas (2002, p.15). 


\section{Aportes del conductismo y constructivismo al diseño curricular e instruccional}

Las dos posturas descritas tienen profundas diferencias, (véase Tabla 3), pero también comparten aspectos en común como lo es: el propósito de mejorar el aprendizaje y el avance del aprendizaje de los estudiantes por etapas o fases. Tanto las diferencias como las semejanzas influyen en la organización de los materiales, en los procesos para la realización de prácticas por parte del estudiante y en la programación de las sesiones de repaso y/o autoevaluación (Schunk, 1997). Desde la posición epistemológica en donde radica la mayor diferencia entre el conductismo y el constructivismo; en el primero imperan los presupuestos objetivos y en el segundo los subjetivos. Mientras que el conductismo motiva la descripción de la realidad, el constructivismo apela a la modelización de un mundo desde la construcción individual o grupal social, cultural y físico (Cattaneo, s.f.) (veáse Tabla 4).

Tabla 3: Diferencias del conductismo y constructivismo

\begin{tabular}{|c|c|c|}
\hline & Conductismo & Constructivismo \\
\hline Diferencias & $\begin{array}{l}\text { Desde la percepción de los } \\
\text { objetivos de la investigación } \\
\text { científica, (positivista) le interesa } \\
\text { la explicación a través de la } \\
\text { predisposición y control de las } \\
\text { variables. } \\
\text { La naturaleza del conocimiento } \\
\text { este formulada por hipótesis } \\
\text { comprobables, verificables } \\
\text { expresadas como leyes y hechos } \\
\text { generalizados y la acumulación } \\
\text { del conocimiento se da por } \\
\text { asociaciones de causa y efecto. } \\
\text { (Velandia, 2006). }\end{array}$ & $\begin{array}{l}\text { - Le interesa por comprensión de } \\
\text { los fenómenos y el desarrollo } \\
\text { humano } \\
\text { Atiende a la construcción y } \\
\text { reconstrucción del conocimiento } \\
\text { investigativo individual, pero } \\
\text { establecido por consenso, con } \\
\text { un mayor ámbito de información } \\
\text { y con posiciones valorativas. } \\
\text { (Velandia, 2006). }\end{array}$ \\
\hline
\end{tabular}

Fuente: Velandia, (2005). 


\section{Tabla 4: Aportes del conductismo al diseño} curricular e instruccional

\begin{tabular}{|c|c|c|c|c|}
\hline $\begin{array}{l}\text { Principios de } \\
\text { aprendizaje }\end{array}$ & Fundamentos & $\begin{array}{l}\text { Al diseño } \\
\text { curricular }\end{array}$ & $\begin{array}{c}\text { Tipos de } \\
\text { aprendizaje }\end{array}$ & $\begin{array}{c}\text { Al diseño } \\
\text { instruccional }\end{array}$ \\
\hline \begin{tabular}{|l|} 
De continuidad: \\
estímulo y \\
respuesta- \\
genera una \\
asociación hasta \\
-convertirse en \\
hábito. \\
La ley del efecto \\
en forma de \\
aprendizaje \\
que se da por \\
estímulo y \\
respuesta \\
(Picado, 2006).
\end{tabular} & \begin{tabular}{|l} 
- \\
La experiencia \\
es el \\
principio del \\
conocimiento. \\
Lo que se \\
aprende es un \\
reflejo de la \\
realidad. \\
El estudiante \\
es un receptor \\
pasivo. \\
No hay en la \\
mente una \\
estructura \\
organizativa. \\
Los estímulos \\
en el ambiente \\
provocan una \\
respuesta \\
(Picado, 2006).
\end{tabular} & $\begin{array}{ll}\text { Condiciones } \\
\text { ambientales del } \\
\text { aprendizaje. } \\
\text { El } \\
\text { conocimiento } \\
\text { puede ser } \\
\text { medible y } \\
\text { evaluado } \\
\text { objetivamente. } \\
\text { Determinación } \\
\text { de los } \\
\text { refuerzos más } \\
\text { apropiados. } \\
\text { En esta misma } \\
\text { secuencia } \\
\text { se espera la } \\
\text { aplicación del } \\
\text { conocimiento } \\
\text { aprendido } \\
\text { a nuevas } \\
\text { situaciones. } \\
\text { Proceso de } \\
\text { aprendizaje } \\
\text { pasivo, deja } \\
\text { un papel } \\
\text { protagonista } \\
\text { al profesor } \\
\text { (Driscoll, 2005 } \\
\text { y Salas, 2002). }\end{array}$ & $\begin{array}{l}\text { Discriminación, } \\
\text { generalización, } \\
\text { asociación y } \\
\text { encadenamiento, } \\
\text { sin llegar a } \\
\text { adquisiciones } \\
\text { de alto nivel } \\
\text { (Driscoll, 2005; } \\
\text { Ertmer \&y Newby, } \\
\text { mencionado por } \\
\text { Schunk 1997). }\end{array}$ & 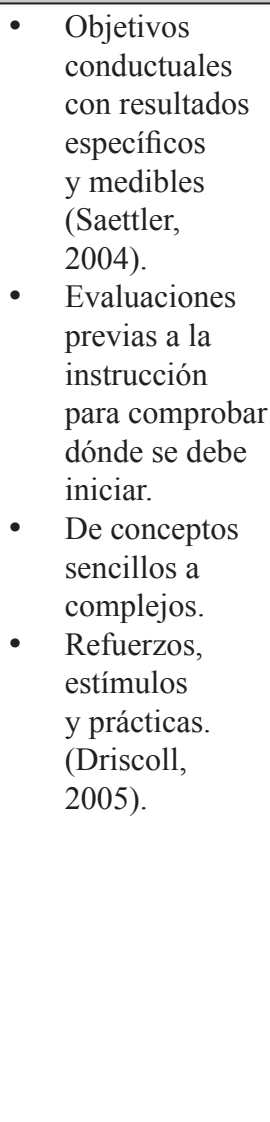 \\
\hline
\end{tabular}

Nota: Adaptación de Driscoll (2005); Picado (2006); Salas (2002) y Schunk (1997). 
Tabla 5: Aportes del constructivismo al diseño curricular y al instruccional

\begin{tabular}{|c|c|c|c|c|}
\hline $\begin{array}{l}\text { Principios del } \\
\text { aprendizaje }\end{array}$ & Fundamentos & $\begin{array}{l}\text { Al diseño } \\
\text { curricular }\end{array}$ & $\begin{array}{c}\text { Tipos de } \\
\text { aprendizaje }\end{array}$ & $\begin{array}{l}\text { Al diseño } \\
\text { curricular }\end{array}$ \\
\hline $\begin{array}{l}\text { Enfoque del } \\
\text { aprendizaje está } \\
\text { centrado en el } \\
\text { estudiante. } \\
\text { Reconoce la } \\
\text { forma del saber } \\
\text { en un sujeto } \\
\text { activo que } \\
\text { interactúa con } \\
\text { otros en un } \\
\text { mundo social, } \\
\text { cultural, físico; } \\
\text { con historia y } \\
\text { conocimientos } \\
\text { previos (López, } \\
\text { s.f.). } \\
\text { Implicaciones } \\
\text { en cómo los } \\
\text { profesores } \\
\text { enseñan y } \\
\text { aprenden a } \\
\text { enseñar? }\end{array}$ & $\begin{array}{l}\text { Equipara el } \\
\text { aprendizaje } \\
\text { con la creación } \\
\text { de nuevos } \\
\text { significados } \\
\text { a partir de un } \\
\text { conocimiento } \\
\text { previo y de las } \\
\text { experiencias. } \\
\text { Promover la } \\
\text { interacción } \\
\text { entre los } \\
\text { factores } \\
\text { ambientales y } \\
\text { el estudiante } \\
\text { (Ertmer } \\
\text { \& Newby, } \\
\text { mencionado } \\
\text { por Schunk } \\
\text { 1997). }\end{array}$ & $\begin{array}{ll}\text { Identificar y } \\
\text { proporcionar } \\
\text { ambientes o } \\
\text { contextos de } \\
\text { aprendizaje. } \\
\text { Interacción con } \\
\text { la información } \\
\text { en diversidad de } \\
\text { presentaciones. } \\
\text { Profesor } \\
\text { como guía o } \\
\text { facilitador. } \\
\text { Interacción } \\
\text { grupal y } \\
\text { tiempos de } \\
\text { reflexión. } \\
\text { Propiciar la } \\
\text { solución de } \\
\text { problemas por } \\
\text { parte de los } \\
\text { estudiantes } \\
\text { Evaluación } \\
\text { enfocada en la } \\
\text { construcción del } \\
\text { conocimientos y } \\
\text { habilidades. } \\
\text { (Driscoll, 2005 } \\
\text { y Hoover (1996) }\end{array}$ & $\begin{array}{l}\text { La construcción } \\
\text { del conocimiento } \\
\text { es que los alumnos } \\
\text { construyen } \\
\text { sus nuevos } \\
\text { conocimientos } \\
\text { utilizando lo que ya } \\
\text { conocen y que el } \\
\text { aprendizaje es activo } \\
\text { (Hoover, 1996). }\end{array}$ & $\begin{array}{l}\text { Creatividad } \\
\text { en cuanto a } \\
\text { actividades } \\
\text { sistemáticas, de } \\
\text { largo plazo y de } \\
\text { reflexión en la } \\
\text { práctica. } \\
\text { Promover el } \\
\text { descubrimiento } \\
\text { de múltiples } \\
\text { perspectivas } \\
\text { (Driscoll, 2005). }\end{array}$ \\
\hline
\end{tabular}

Fuentes: Driscoll, (2005); Hoover, (1996); López, (s.f) y Schunk, (1997).

\section{Conductismo y Constructivismo en el Proceso de Enseñanza y Aprendizaje de las Ciencias Naturales}

Perdomo (2007), desde el paradigma socio-crítico, ve la necesidad de asumir el asunto de la naturaleza y los recursos desde una visión holística. Un proceso de construcción-reconstrucción de la nueva realidad, representado por el constructivismo en las teorías del aprendizaje.

Desde el interés científico de las ciencias naturales se esperarían diseños curriculares con calidad en el proceso de la generación del conocimiento tomando en cuenta la racionalidad procedimental tal como lo establece Simon (citado por Ramos, 2003). 
Es entonces pertinente que la construcción y validación de las estrategias de enseñanza y aprendizaje en el diseño curricular cuenten con la participación representativa de la población interdisciplinaria académica, docente y estudiantil involucrada, tal y como lo indica el enfoque de la ciencia post normal (Funtowicz y Ravetz, 2000).

Tovar (2008), invita a plantear iniciativas desde la dinámica de las Ciencias Naturales para proponer el aprendizaje, la enseñanza y la evaluación de este tipo de temática ambiental, con dimensiones conceptuales, metodológicas, actitudinales y comunicativas ante contextos políticos, ambientales, económicos y culturales. Es así que en el diseño curricular los aprendizajes, se deben integrar la resolución de presentes y futuros problemas con objetivos el cambio de actitud, habilidades técnicas en el manejo de alguna especie, identificación de procesos.

El conductismo y el constructivismo son posiciones presentes en la enseñanza de varias áreas del conocimiento, entre ellas se puede mencionar el de las ciencias naturales. Campo del saber en el que se observa un predominio del enfoque positivista y post positivista, a lo que Capra, (mencionado por Morán, 2003), llama "la adopción, en los últimos trescientos años, del paradigma universal cartesiano” (p.7).

En la investigación científica en el área de las Ciencias Naturales ha predominado de acuerdo con Cerda (2000), varios métodos para acercarse al objeto de estudio, la postulación de la inducción, de la experimentación y del análisis y la síntesis en actividades prácticas de campo.

Sin embargo, a partir del nacimiento del movimiento constructivista en los procesos de enseñanza, se asume desde la pedagogía de las ciencias naturales una epistemología que va dirigida a la construcción del conocimiento, especialmente con el uso de actividades en donde se rescata la experiencia del estudiante tanto en el aula como en el contexto natural, social y cultural que lo rodea. De tal manera, que se logra que el estudiante aplique la práctica como estrategia de aprendizaje en un intercambio horizontal en actividades planeadas desde el diseño instruccional como las giras de campo y la interacción con diferentes actores sociales.

Es entonces, evidente que en el proceso de enseñanza de las Ciencias Naturales se plantea la práctica como actividad predominante. Este tipo de ejercicios lo definen López, Arévalo, Rodríguez, Andrade-Piedra y Quirós (2008) como 
una herramienta que facilita el conocimiento vivencial a través de visitas a experiencias. Sobre las que interesa entender su desarrollo, resultados y principales lecciones aprendidas, con el propósito de promover el intercambio horizontal de conocimientos entre distintos actores en un área específica y motivar el interés para la réplica de experiencias similares. (p.1).

Las experiencias vivenciales contemplan la combinación de elementos teórico-prácticos y la participación de los actores directo en las experiencias o visitas en los cuales se genera el intercambio de conocimientos, análisis y reflexión.

Los cambios en los enfoques de las actividades en la enseñanza de las Ciencias Naturales conforman una transición del paradigma basado en el pensamiento científico cartesiano a un paradigma holístico y multidisciplinario (Morán, 2003). En dichas variaciones se puede apreciar principios del constructivismo en el cual se vincula también la interdisciplinariedad. Tal como se observa en la Figura 3, el constructivismo reúne un conjunto de saberes, en donde todo conocimiento, contexto, disciplina está en relación con el desarrollo en el proceso de construcción del saber, el conocimiento de las partes para la comprensión del todo.

Sea bajo un enfoque conductivista o constructivista, las actividades vivenciales experimentales o no, requieren de una planeación que garantice el aprovechamiento de los estudiantes en su proceso de aprendizaje. Es a través del diseño instruccional, entendiéndolo como un serie de etapas sistemáticas que maximizan el aprendizaje de un método, una técnica, una materia, una lección, un tema, una actividad y materiales a desarrollar (Brigss, Gustafson y Tillman; 1991 y Yukavestlky, 2003). 
Figura 3: Adaptado del conjunto de saberes de Fritjof Capra (2003)

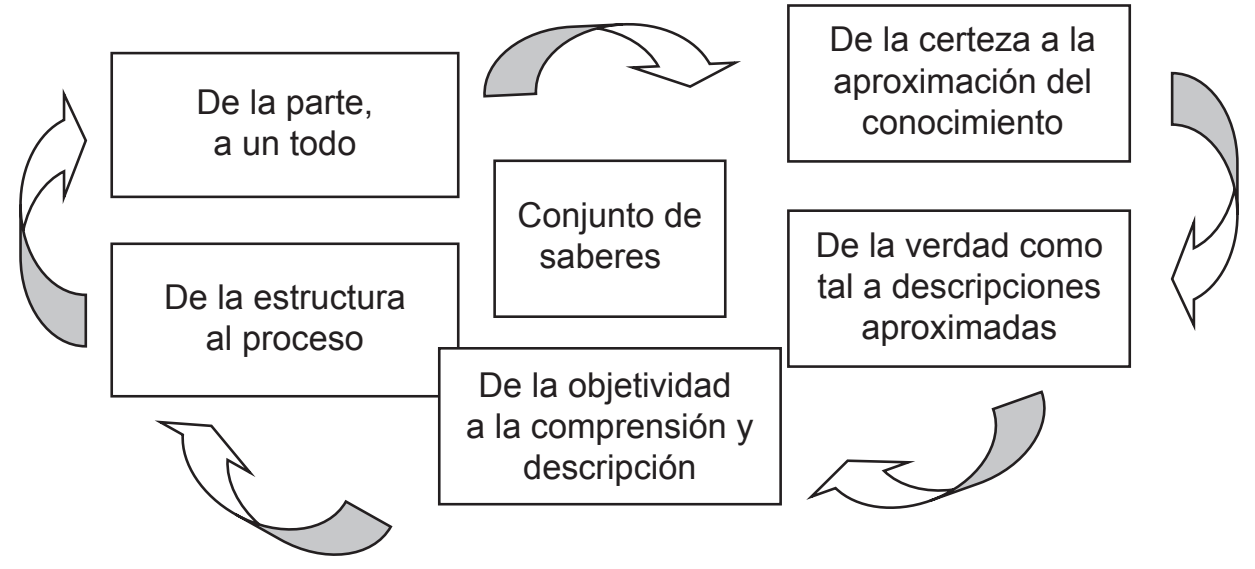

\section{Conclusión}

Se puede indicar que hay una serie de aspectos curriculares, y de diseño instruccional, que deben ser implementados, integrando las aportaciones del conductismo en los primeros cursos de un plan de estudios, para ir desarrollando un enfoque constructivista en los subsiguientes, que permita al estudiante un autoaprendizaje en su propia construcción del conocimiento.

En los procesos de enseñanza y aprendizaje, se deberá tener en cuenta desde el diseño curricular el paradigma dominante que impere en el estudio del área del conocimiento, así como su correspondencia con el planeamiento didáctico desde la teoría (as) de aprendizaje que promuevan las etapas necesarias de desarrollo cognitivo en los estudiantes a través de sus estudios. El diseño instruccional ofrece el aseguramiento de la planeación en cualquiera de las necesidades didácticas del proceso de enseñanza y aprendizaje, especialmente en aquellas actividades vivenciales, sean individuales o participativas.

Se puede indicar que en las Ciencias Naturales y en los recursos naturales, se conjuga una posición positivista en la investigación y una constructivista subjetiva en la pedagogía. La diversidad de los enfoques paradigmáticos de investigación y de las teorías de aprendizaje, permite el uso de métodos inductivos, deductivos y analíticos, con los cuales se busca la formulación integral que responda a los objetivos completos y pertinentes como respuesta a las necesidades de formación profesional, de ciudadanos que incorporen su conocimiento, habilidades y compromiso a la solución de la problemática actual y en contextos reales. 


\section{Referencias bibliográficas}

Briggs, L. Gustafson, K. y Tillman, M. (1991). Instruccional design: Principles an application ( $2^{\mathrm{a}}$ ed.). USA.: Educational Technology Publications.

Capra, F. (2003). Las Conexiones ocultas: Implicaciones sociales, medioambientales, económicas y biológicas de una nueva visión del mundo. España: Editorial Anagrama.

Cattaneo, M. (s.f.). Teorías educativas contemporáneas y modelos de aprendizaje. Argentina: Universidad de Palermo.

Cerda, H. (2000). La investigación total. Bogotá: El búho.

Claude, J. (2008). Epistemología ética y ciencia de la educación. Argentina: Encuentro Grupo Editor.

De Zubiría, J. (2008). De la escuela nueva al constructivismo: Un análisis crítico. (2a Ed.) Colombia: Cooperativa Editorial Magisterio.

Driscoll, M. (2005). Psychology of learning for instruction (3 ${ }^{\text {rd }}$ Ed.). EE.UU.: Pearson Education. Inc.

Funtowicz, S. y Ravetz, J. (2000). La ciencia posnormal: Ciencias con la Gente. (1a ed.) Barcelona. España: Icaria 2000.

Guba, E. (1990). The paradigm dialog. Newbury Park, Ca. EE.UU.: Sage Publications, Inc.

Hernández, P. (2008). El Paradigma Conductista y su Aplicación en el Proceso Educativo 1,2 y 3. Recuperado de http://www.espaciologopedico.com/articulos/ articulos2.php?Id_articulo $=1557$

Hoover, W. (1996). The Practice Implications of Constructivism. Constructivism (9). 3. SEDL Letter. Recuperado de http://www.sedl.org/pubs/sedletter/v09n03/practice.html

López, G., Arévalo, D., Rodríguez, F., Andrade-Piedra, J. y Quirós, C. (2008). Gira de aprendizaje: Enfoque participativo de cadenas productivas. EPCP. Recuperado de www.cambioandino.net/aca/c/document_library/get_file?folderId=85\&name $=$ DLFE- 45. doc

López, R. (s.f.). Idea de constructivismo. Revista Praxis (5). Recuperado de http:// www.udp.cl/psicologia/publicaciones/praxis.htm

Martín, L. (1991). Diez Lecciones de epistemología. España: Editorial Akal, S.A.

Moran, H. (2003). Ciencia económica, del paradigma cartesiano. Revista de la Facultad de Ciencias Económicas (4),14, 154-171. Recuperado de http://sisbib.unmsm. edu.pe/BibVirtualData/publicaciones/economia/14/pdf/ciencia_economia.pdf

Perdomo, M.E. (2007). El Problema Ambiental: hacia una interacción de las ciencias naturales y sociales Revista Iberoamericana de Educación. 44/3. Organización de Estados Iberoamericanos para la Educación, la Ciencia y la Cultura (OEI). Recuperado de http://www.rieoei.org/deloslectores/1898Lopez.pdf

Picado, F.M. (2006). Didáctica general. Una perspectiva ntegrada (8a. Ed.). San José, Costa Rica: EUNED.

Ramos, J. (2003). La Perspectiva biofísica del proceso económico: Economía ecológica. En Falconi, F., Hercowitz, H. y Muradiam, R. (2004). (Eds.), Globalización y Desarrollo en América Latina (pp. 19-47). Ecuador: FLACSO. 
Salas, F. E. (2002). Epistemología, educación y tecnología educativa. Educación. Vol. 26, 001, 9-18.

Soto, J.A. y Bernardini, A. (2007). La educación actual en sus fuentes filosóficas. San José, Costa Rica: EUNED.

Schunk, D. (1997). Teoría del aprendizaje (2 $2^{\mathrm{a}}$ ed.). México: Prentice Hall.

Thanasoulas, D. (2002). Constructivist learning. Karen's Linguistics Issues. Recuperado de http://www3.telus.net/linguisticsissues/constructivist.html

Tovar, J.C. (2008). Propuesta de modelo de evaluación multidemensional de los aprendizajes en ciencias naturales y su relación con la estructura de la didáctica de las ciencias. Revista Eureka sobre Enseñanza y Divulgación de las Ciencias. 003, 259-273.

Velandia, M.A. (2006). Estrategias para construir la convivencia solidaria en el aula universitaria: Trabajos en equipo y comunicación generadora de mundos. Colombia: Universidad Cooperativa de Colombia. Facultad de Educación.

Yukavestsky, G. (2003). La elaboración de un modelo instruccional. Recuperado de http://academic.uprm.edu/_marion/tecnofilia2011/files/1277/CCC_LEDUMI. pdg 